\title{
INVARIANT THEORY AND THE LAMBDA ALGEBRA
}

\author{
BY
}

WILLIAM M. SINGER ${ }^{1}$

\begin{abstract}
Let $A$ be the Steenrod algebra over the field $F_{2}$. In this paper we construct for any left $A$-module $M$ a chain complex whose homology groups are isomorphic to the groups $\operatorname{Tor}_{s}^{A}\left(F_{2}, M\right)$. This chain complex in homological degree $s$ is built from a ring of invariants associated with the action of the linear group $G L_{s}\left(F_{2}\right)$ on a certain algebra of Laurent series. Thus, the homology of the Steenrod algebra (and so the Adams spectral sequence for spheres) is seen to have a close relationship to invariant theory. A key observation in our work is that the Adem relations can be described in terms of the invariant theory of $G_{\nu_{2}}\left(F_{2}\right)$. Our chain complex is not new: it turns out to be isomorphic to the one constructed by Kan and his coworkers from the dual of the lambda algebra. Thus, one effect of our work is to give an invariant-theoretic interpretation of the lambda algebra. As a consequence we find that the dual of lambda supports an action of the Steenrod algebra that commutes with the differential. The differential itself appears as a kind of "residue map". We are also able to describe the coalgebra structure of the dual of lambda using our invariant-theoretic language.
\end{abstract}

1. Introduction. Our object in this paper is to use classical invariant theory to describe a chain complex, from which one can compute the "homology groups of the Steenrod algebra", that is, the groups $\operatorname{Tor}_{s}^{A}\left(F_{2}, M\right)$, for an arbitrary left $A$-module $M$. Our work provides an invariant-theoretic description of the "lambda algebra", introduced by the six authors of [1]. We show how, as a consequence of our work, we can endow lambda in each homological degree with an action of the Steenrod algebra that commutes with the differential.

In more detail, let $P_{s}=F_{2}\left[t_{1}, \ldots, t_{s}\right]$ be the polynomial algebra over $F_{2}$ on generators $t_{i}$ of dimension one. The linear group $G L_{s} \equiv G L_{s}\left(F_{2}\right)$ acts on $P_{s}$ as a group of algebra automorphisms, and the invariant ring $P_{s}^{G L_{s}}$, has been computed by Dickson [3]. He found it to be a polynomial algebra on certain generators $Q_{s, i}$ of dimension $2^{s}-2^{i}$ :

$$
P_{s}^{G L, s}=F_{2}\left[Q_{s, 0}, Q_{s, 1}, \ldots, Q_{s, s-1}\right]
$$

For applications to the Steenrod algebra we introduce a ring of invariants somewhat larger than (1.1). Let $\Phi_{s}$ be the algebra obtained from $P_{s}$ by inverting all

Received by the editors February 25, 1982 and, in revised form, November 4, 1982.

1980 Mathematics Subject Classification. Primary 55Q45, 55S10, 55U10, 55T15.

Key words and phrases. Adams spectral sequence, homotopy groups of spheres, lower central series, lambda algebra, cohomology of the Steenrod algebra.

'Research partially supported by National Science Foundation research grant MCS 8101702. 
nonzero linear forms in the variables $t_{i}$; that is, all elements of the type

$$
\left\{\sum_{i=1}^{s} \lambda_{i} t_{i} \mid \lambda_{i} \in F_{2} ; \lambda_{1} \neq 0 \text { for at least one } i\right\} \text {. }
$$

Then $G L_{s}$ acts on $\Phi_{s}$ as a group of algebra automorphisms. We will define a certain $G L_{s}$-subalgebra $\Phi_{s}^{+} \subset \Phi_{s}$ consisting of elements whose denominators are "not too large", in a sense that we make precise in $\S 2$. We write

$$
\Gamma_{s}^{+}=\left(\Phi_{s}^{+}\right)^{G L_{*}}
$$

for the ring of invariants. Like (1.1), this ring can easily be described in terms of the generators $Q_{s, i}$. We need only let $Q_{s, 0}$ appear with negative exponents (see Proposition 2.4).

The main work of this paper is to associate to any left $A$-module $M$ a certain chain complex $\Gamma^{+} M$. As a vector space,

$$
\left(\Gamma^{+} M\right)_{s}=\Phi_{s}^{+} \otimes M .
$$

The most important property of the chain complex is given by

THEOREM 1.1. There is a natural isomorphism

$$
\operatorname{Tor}_{s}^{A}\left(F_{2}, M\right)=H_{s}\left(\Gamma^{+} M\right) \text {. }
$$

Thus, the "homology groups of the Steenrod algebra" are seen to be closely related to the invariant theory of the general linear groups. These results are all obtained in the first four sections of the paper.

Having said this we must confess that only the invariant-theoretic description of our chain complex $\Gamma^{+} M$ is new; the complex itself is not. $\Gamma^{+} M$ is just the dual of the cochain complex constructed in [1] from the "lambda algebra". In particular if $M=F_{2}$ we will show how to give $\Gamma^{+} F_{2}$ the structure of a differential coalgebra, in such a way that the following theorem holds.

THEOREM 1.2. Let $\Lambda^{*}$ be the dual of $\Lambda$. Then there is an isomorphism of differential coalgebras

$$
\Gamma^{+} F_{2} \simeq \Lambda^{*} .
$$

We demonstrate this in $\S 8$. The effect of our work is thus to give an invariant theoretic interpretation of the lambda algebra.

A close relationship between the Adem relations and the invariant theory of $G L_{2}$ is central to the construction of $\Gamma^{+} M$, and to the isomorphism (1.6). We describe this connection in $\S 3$, and use it prove our main theorems.

By recasting the lambda algebra in invariant-theoretic language, we discover some heretofore unsuspected properties of its dual. We show in $\$ 5$ :

Theorem 1.3. Let $M$ be a graded left A-module. For each $s \geqslant 0,\left(\Gamma^{+} M\right)_{s}$ has a natural left $A$-action, in such a way that the differential $\mathrm{\partial}:\left(\Gamma^{+} M\right)_{s} \rightarrow\left(\Gamma^{+} M\right)_{s-1}$ is an A-linear map. The induced A-action on $\operatorname{Tor}_{s}^{A}\left(F_{2}, M\right)=H_{s}\left(\Gamma^{+} M\right)$ is trivial.

This result gives an interesting interpretation of the differential. The $A$-action on $\left(\Gamma^{+} M\right)_{s}$ is very rich. The differential kills it. 
To describe the $A$-action on $\left(\Gamma^{+} M\right)_{s}$ in general requires that we use the main construction of [8]; but in the special case $M=F_{2}$ the action on $\left(\Gamma^{+} F_{2}\right)_{s}=\mathrm{I}_{s}^{+}$is easily described. In fact the polynomial algebra $P_{s}$ acquires an $A$-action from its identification with the cohomology ring $H^{*}\left(R P^{\infty} \times R P^{\infty} \times \cdots \times R P^{\infty}\right)$. This action extends uniquely to an action on the localization $\Phi_{s}^{+}$(in the manner of [11, Proposition 2.1]) which commutes with the action of $G L_{s}$. Consequently, $\Gamma_{s}^{+}=\left(\Phi_{s}^{+}\right)^{G L_{s}}$ acquires the structure of an algebra over $A$, and the action on $\left(\Gamma^{+} M\right)_{s}$ described in Theorem 1.3 reduces to this one in the case $M=F_{2}$.

As another corollary of our invariant-theoretic description of the lambda algebra, we find that the differential on $\Lambda^{*}$ can be described in an interesting way. In fact, we show in $\S 9$ that $\partial: \Gamma_{s}^{+} \rightarrow \Gamma_{s-1}^{+}$is a kind of residue map. Given $\gamma \in \Gamma_{s}^{+}$, one expands $\gamma$ (a rational function in $t_{1}, t_{2}, \ldots, t_{s}$ ) as a Laurent series about the origin of the $t_{s}$-plane. Then $\partial \gamma$ is the coefficient of $t_{s}^{-1}$ in the series expansion. We express this result in the form

THEOREM 1.4. Let $\gamma \in \Gamma_{s}^{+}=\left(\Gamma^{+} F_{2}\right)_{s}$ be given. Then

$$
\partial \gamma=\operatorname{Res} \gamma \text {. }
$$

Care has been taken to make this paper reasonably selfcontained. In the first four sections we carry out from scratch the construction of $\Gamma^{+} M$ and the proof of Theorem 1.1. Comparison with the lambda algebra and the proof of Theorem 1.2 are postponed to $\$ \S 6-8$, and even there prior knowledge of the lambda algebra is not assumed. The proof of Theorem 1.4 is contained in $\$ 9$. It, too, can be understood entirely within the context of the present paper. Only in $\$ 5$, where Theorem 1.3 is proved, must we summarize and use the main results of [8].

Although this paper is independent of [9], I express my warmest thanks to William Dwyer and Haynes Miller for pointing out the relationship between the constructions of [9] and the lambda algebra, thus giving the current paper its start. I thank Clarence Wilkerson for suggesting that invariant rings should play a role, and for his help in understanding the coproduct in $\Gamma^{+} F_{2}$. The elegant definition (6.4) of the differential in the lambda algebra was shown to me by Peter Bousfield and Stewart Priddy.

2. Invariant theory. In this section we collect those results from the theory of invariants that we will need to describe the chain complexes $\Gamma^{+} M$.

Write $P_{s}=F_{2}\left[t_{1}, \ldots, t_{s}\right]$ for the polynomial algebra over $F_{2}$ on $s$ generators, each of dimension one. The group $G L_{s} \equiv G L_{s}\left(F_{2}\right)$ acts naturally on the vector space of one-dimensional elements of $P_{s}$. We extend this action to the full polynomial ring by requiring $G L_{s}$ to act as a group of algebra automorphisms.

Let $T_{s} \subseteq G L_{s}$ be the subgroup consisting of all upper triangular matrices with "ones" on the main diagonal. The invariant ring $P_{s}^{T_{s}}$ has been determined by Mui [7, Theorem 3.4]. He finds that $P_{s}^{T_{s}}$ is a polynomial algebra

$$
P_{s}^{T_{s}}=F_{2}\left[V_{1}, V_{2}, \ldots, V_{s}\right]
$$

on elements $V_{k}$ of dimension $2^{k-1}$. $V_{k}$ is given by

$$
V_{k}=\prod_{\lambda}\left(\lambda_{1} t_{1}+\cdots+\lambda_{k-1} t_{k-1}+t_{k}\right)
$$


where the product is over all $(k-1)$-tuples $\lambda=\left(\lambda_{1}, \ldots, \lambda_{k-1}\right)$ with $\lambda_{i} \in F_{2}$.

The invariant ring $P_{s}^{G L_{\text {s }}}$ has been determined by Dickson [3, pp. 76, 80]. He finds that $P_{s}^{G L_{s}}$ is also a polynomial algebra

$$
P_{s}^{G L,}=F_{2}\left[Q_{s, 0}, Q_{s, 1}, \ldots, Q_{s, s-1}\right]
$$

on generators $Q_{s, i}$ of dimension $2^{s}-2^{i}$. The element we call $Q_{s .0}$ is called $L_{s}$ by Dickson, it is given by

$$
Q_{s .0}=\prod_{\lambda}\left(\lambda_{1} t_{1}+\cdots+\lambda_{s} t_{s}\right)
$$

where the product is over all $s$-tuples $\lambda=\left(\lambda_{1}, \ldots, \lambda_{s}\right)$ with $\lambda_{i} \in F_{2}$, for which $\lambda \neq(0,0, \ldots, 0)$. Clearly,

$$
Q_{s, 0}=V_{1} V_{2} \cdots V_{s} \text {. }
$$

The elements $Q_{s, i}$ for $0<i<s$ are a little harder to describe. Dickson gives $Q_{s, i}$ as a quotient $Q_{s, i}=[0,1, \ldots, \hat{i}, \ldots, s] / Q_{s, 0}$, where $[0,1, \ldots, \hat{i}, \ldots, s]$ is a certain determinant in the variables $t_{i}[3, \mathrm{p} .75]$. Of course one also has $P_{s}^{G L_{s}} \subseteq P_{s}^{T_{s}}$, so one can expect a formula for $Q_{s, i}$ in terms of the $V_{k}$ of (2.2). A formula of this kind is given by Dickson: he describes $Q_{s . i}$ inductively in $s$ by proving

$$
Q_{s, i}=V_{s} \cdot Q_{s-1, i}+Q_{s-1, i-1}^{2} \quad(0 \leqslant i<s) .
$$

This is [3, equation (4), p. 80]. To interpret (2.6) read $Q_{s-1, s-1}=1$ if $s \geqslant 1$, and $Q_{s, i}=0$ if $i<0$ or $i>s$. Then (2.6) determines the $Q_{s, i}$, and the reader may take it as the definition if he wishes. For example, $Q_{1,0}=V_{1}=t_{1} ; Q_{2.0}=V_{1} V_{2}=t_{1}^{2} t_{2}+$ $t_{1} t_{2}^{2} ; Q_{2.1}=V_{1}^{2}+V_{2}=t_{1}^{2}+t_{1} t_{2}+t_{2}^{2}$. Notice that (2.6) implies (2.5).

For our applications to the lambda algebra we will need analogues of (2.1) and (2.3) for the case in which the polynomial algebra $P_{s}$ is replaced by an algebra of rational functions in the $t_{i}$. In fact, let $S(s) \subseteq P_{s}$ be the multiplicative subset generated by all the one-dimensional classes in (1.2). Let $\Phi_{s}$ be the localization

$$
\Phi_{s}=\left(P_{s}\right)_{S(s)}
$$

as in $\S 1$. Then $G L_{s}$ acts on $\Phi_{s}$ as a group of algebra automorphisms. We write $\Delta_{s}=\left(\Phi_{s}\right)^{T_{s}}$ and $\Gamma_{s}=\left(\Phi_{s}\right)^{G L_{s}}$ for the invariant rings for each $s \geqslant 1$; and we define $\Delta_{0}=\Gamma_{0}=F_{2}$. Then from (2.1), (2.3) and (2.4) we obtain easily for each $s \geqslant 1$

$$
\begin{gathered}
\Delta_{s}=\left(\Phi_{s}\right)^{T_{s}}=F_{2}\left[V_{1}^{ \pm 1}, V_{2}^{ \pm 1}, \ldots, V_{s}^{ \pm 1}\right], \\
\Gamma_{s}=\left(\Phi_{s}\right)^{G L_{s}}=F_{2}\left[Q_{s, 0}^{ \pm 1}, Q_{s, 1}, \ldots, Q_{s, s-1}\right] .
\end{gathered}
$$

We will need an alternate set of generators for $\Delta_{s}$. Set

$$
v_{1}=V_{1}, \quad v_{k}=V_{k} / V_{1} V_{2} \cdots V_{k-1} \quad(k \geqslant 2)
$$

so that

$$
V_{k}=v_{1}^{2^{k-2}} v_{2}^{2^{k-3}} \cdots v_{k-1} v_{k} \quad(k \geqslant 2) .
$$

Then (2.8) could just as well be written

$$
\Delta_{s}=\left(\Phi_{s}\right)^{T_{s}}=F_{2}\left[v_{1}^{ \pm 1}, v_{2}^{ \pm 1}, \ldots, v_{s}^{ \pm 1}\right] \text {. }
$$


The new generators will be useful in constructing the chain complexes $\Gamma^{+} M$, and in relating $\Gamma^{+} F_{2}$ to the dual of lambda (see (7.1)). Note that $\operatorname{dim} v_{k}=1$ for all $k$.

We will use our new generators to make a coalgebra from the spaces $\Delta_{s}$. In fact, for any pair of nonnegative integers $p, q$, for which $p+q=s$ we define an algebra isomorphism $\psi_{p, q}: \Delta_{s} \rightarrow \Delta_{p} \otimes \Delta_{q}$ by

$$
\psi_{p . q}\left(v_{i}\right)= \begin{cases}v_{i} \otimes 1, & 1 \leqslant i \leqslant p, \\ 1 \otimes v_{i-p}, & p+1 \leqslant i \leqslant s .\end{cases}
$$

Here we understand $\Delta_{0}=F_{2} ; \psi_{s, 0}(x)=x \otimes 1 ; \psi_{0, s}(x)=1 \otimes x$. If we now set $\Delta=\bigoplus_{s \geqslant 0}\left(\Delta_{s}\right)$ then the maps (2.13) combine to define a coproduct $\psi: \Delta \rightarrow \Delta \otimes \Delta$ under which $\Delta$ becomes a connected, graded coalgebra.

Similarly let us write $\Gamma=\bigoplus_{s \geqslant 0}\left(\Gamma_{s}\right)$. We would like to show that $\Gamma$ is a subcoalgebra of $\Delta$. To this end, we assert that if $p, q \geqslant 0$ and $p+q=s$ then

$$
\psi_{p, q}\left(Q_{s, i}\right)=\sum_{j \geqslant 0} Q_{p, 0}^{2^{4}-2^{\prime}} Q_{p, i-j}^{2^{\prime}} \otimes Q_{q, j}
$$

for each $i, 0 \leqslant i<s$. (The conventions of (2.6) are in force here as well: $Q_{i, i}=1$ if $i \geqslant 0 ; Q_{s, i}=0$ if $i<0$ or $i>s$.) The proof of (2.14) is an easy induction on $s$ : one uses $(2.5),(2.6),(2.11)$, and the fact that $\psi_{p, q}$ preserves products. The reader may wish to compare (2.14) to Madsen's formula for the diagonal in the dual of the Dyer-Lashof algebra; see Proposition 3.4 of [5]. The formulas are identical.

Since $\psi_{p, q}$ is a map of algebras, (2.14) determines $\psi_{p, q}$ on $\Gamma_{s}$. We have at once

PROPOSITION 2.1. $\psi_{p, q}\left(\Gamma_{s}\right) \subseteq \Gamma_{p} \otimes \Gamma_{q}$; so $\Gamma$ is a subcoalgebra of $\Delta$.

In order to construct the chain complexes $\Gamma^{+} M$, we need to single out a certain subspace of $\Gamma_{s}$ consisting of those $G L_{s}$-invariant rational functions in the variables $t_{i}$ whose denominators are "not too large". We make this notion precise in the following way. An element $\sigma$ of the multiplicative subset $S(s)$ can be written uniquely up to order in the form $\sigma=\sigma_{1}^{i_{1}} \cdots \sigma_{k}^{i_{k}}$, where $i_{1}, \ldots, i_{k} \geqslant 0$ and $\sigma_{1}, \ldots, \sigma_{k}$ are distinct one-dimensional elements of $P_{s}$. Define the "weight" of $\sigma$, written $\rho(\sigma)$, by setting $\rho(\sigma)=\max \left(i_{1}, \ldots, i_{k}\right)$. Now define the subalgebra $\Phi_{s}^{+} \subseteq \Phi_{s}$ by

$$
\Phi_{s}^{+}=\operatorname{Span}\left\{\begin{array}{l|l}
\gamma=\beta / \sigma & \begin{array}{l}
\beta \in P_{s}, \sigma \in S(s) \\
\rho(\sigma) \leqslant \operatorname{dim} \gamma
\end{array}
\end{array}\right\}
$$

for all $s \geqslant 1$; and if $s=0$ write $\Phi_{0}^{+}=F_{2}$. Note in particular that all elements of $\Phi_{s}^{+}$ have nonnegative dimension. A useful lemma in working with $\Phi_{s}^{+}$is

LEMMA 2.2. Let $s \geqslant 1$. If $\gamma \in \Phi_{s}^{+}$is a homogeneous element then it can be written

$$
\gamma=\beta / Q_{s, 0}^{n}
$$

where $\beta \in P_{s}$ and $n$ is an integer for which $0 \leqslant n \leqslant \operatorname{dim} \gamma$.

This is an easy consequence of (2.4) and (2.15).

Clearly the subalgebra $\Phi_{s}^{+} \subseteq \Phi_{s}$ is mapped to itself by the elements of $G L_{s}$. Write

$$
\Delta_{s}^{+}=\left(\Phi_{s}^{+}\right)^{T_{s}}, \quad \Gamma_{s}^{+}=\left(\Phi_{s}^{+}\right)^{G L_{s}}
$$


for the invariant rings if $s \geqslant 1$; and $\Delta_{0}^{+}=\Gamma_{0}^{+}=F_{2}$. Then $\Delta_{s}^{+}$and $\Gamma_{s}^{+}$are subalgebras of $\Delta_{s}$ and $\Gamma_{s}$, respectively. Using Lemma 2.2 we can easily describe monomial bases for these subalgebras.

PROPOSITION 2.3. If $\gamma \in \Delta_{s}^{+}$then $\gamma$ can be written as a sum of monomials $v_{1}^{j_{1}} \cdots v_{s}^{j_{1}}$, each of which lies in $\Delta_{s}^{+}$.

Proposition 2.4. A basis for $\Gamma_{s}^{+}$is given by the monomials

$$
\gamma=Q_{s, 0}^{i_{0}} Q_{s, 1}^{i_{1}} \cdots Q_{s, s-1}^{i_{s,-1}}
$$

for which

$$
-\infty<i_{0}<\infty ; \quad 0 \leqslant i_{1}, i_{2}, \ldots, i_{s-1}
$$

and $0 \leqslant i_{0}+\operatorname{dim} \gamma$.

The proofs are easy. We will show in $\S 8$ that $\Gamma_{s}^{+}$is isomorphic to $\left(\Lambda^{s}\right)^{*}$.

Write $\Gamma^{+}=\bigoplus_{s \geqslant 0}\left(\Gamma_{s}^{+}\right)$. We will also show in $\S 8$ how to regard $\Gamma^{+}$as a quotient coalgebra of $\Gamma$. Note that $\Gamma^{+}$is not a subcoalgebra of $\Gamma$ (compute $\psi_{1,1}\left(Q_{2,0}^{-1} Q_{2,1}^{2}\right)$ ). It is nevertheless true that

$$
\psi_{s-1,1}\left(\Gamma_{s}^{+}\right) \subseteq \Gamma_{s-1}^{+} \otimes \Gamma_{1}
$$

and we will need to know this in $\S 4$. To prove (2.20) take a typical monomial $\gamma \in \Gamma_{s}^{+}$, given by (2.18). Into this expression for $\gamma$ substitute (2.6) in the form $Q_{s, j}=Q_{s-1.0} Q_{s-1, j} v_{s}+Q_{s-1, j-1}^{2}$. We obtain an expansion of $\gamma$ as a sum of

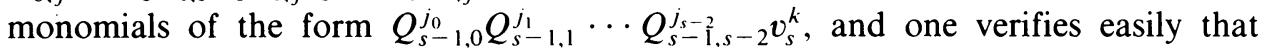
$0 \leqslant j_{0}+\operatorname{dim} \gamma-k$ in every term that appears. This proves $(2.20)$.

As a corollary we obtain

Lemma 2.5. Let $\gamma$ in $\Gamma_{s}^{+}$be given; let

$$
\gamma=\sum v_{1}^{j_{1}} \cdots v_{s}^{j_{s}}
$$

be its expansion in terms of $v_{1}, \ldots, v_{s}$. Then $j_{1} \geqslant 0$ in every monomial that appears.

This is proved by applying (2.20) repeatedly.

The reader who is interested only in the construction $\Gamma^{+} M$ has already read enough invariant theory, and can proceed to $\S \S 3-5$. But in order to compare $\Gamma^{+} F_{2}$ to the dual of $\Lambda$ we will need in $\S 8$ two technical lemmas (Lemmas 2.6 and 2.7 below) that relate the basis for $\Gamma_{s}^{+}$given by Proposition 2.4 to the basis for $\Delta_{s}^{+}$given by Proposition 2.3. In order to state this lemma, we write $I_{s}$ for the set of $s$-tuples of integers $\left(i_{0}, \ldots, i_{s-1}\right)$ for which

$$
0 \leqslant i_{1}, i_{2}, \ldots, i_{s-1} \text { and } 0 \leqslant i_{0}+\sum_{k=0}^{s-1}\left(2^{s}-2^{k}\right) i_{k} .
$$

Then the set $I_{s}$ labels the basis for $\Gamma_{s}^{+}$that is given in Proposition 2.4. We write $J_{s}$ for the set of $s$-tuples of integers $\left(j_{1}, \ldots, j_{s}\right)$ for which

$$
0 \leqslant j_{1} \text { and } j_{k-1} \leqslant 2 j_{k} \quad(2 \leqslant k \leqslant s) \text {. }
$$


In $\S 8$ we will use the fact that $J_{s}$ labels a basis for $\Lambda^{s}$. Define a function $\phi: I_{s} \rightarrow J_{s}$ by writing $\phi\left(i_{0}, \ldots, i_{s-1}\right)=\left(j_{1}, j_{2}, \ldots, j_{s}\right)$ where

$$
j_{k}=2^{s-k}\left(i_{0}+i_{1}+\cdots+i_{k-1}\right)+\sum_{l=0}^{s-k-1}\left(2^{s-k}-2^{l}\right) i_{k+l} .
$$

Define a function $\psi: J_{s} \rightarrow I_{s}$ by writing $\psi\left(j_{1}, \ldots, j_{s}\right)=\left(i_{0}, \ldots, i_{s-1}\right)$ where

$$
i_{0}=j_{1}-j_{2}-\cdots-j_{s}, \quad i_{k-1}=2 j_{k}-j_{k-1} \quad(k \geqslant 2) .
$$

Routine checking shows

LEMMA 2.6. $\phi: I_{s} \rightarrow J_{s}$ is a bijection and $\psi$ is its inverse.

To obtain further information about the basis in Proposition 2.4, let us take as a basis for $\Delta_{s}$ the set of monomials $\left\{v_{1}^{j_{1}} \cdots v_{s}^{j_{s}} \mid j_{1}, \ldots, j_{s} \in Z\right\}$, and order this basis antilexicographically. Simple inductive computations using (2.6) and (2.11) show

Lemma 2.7. Let $\left(j_{1}, \ldots, j_{s}\right)=\phi\left(i_{0}, \ldots, i_{s-1}\right)$. Then in $\Delta_{s}$

$$
Q_{s, 0}^{i_{0}} Q_{s, 1}^{i_{1}} \cdots Q_{s, s-1}^{i_{s}, 1}=v_{1}^{j_{1}} \cdots v_{s}^{j_{s}}+\text { smaller monomials. }
$$

This lemma will help us in $\S 8$ to show $\Gamma_{s}^{+}$isomorphic to $\left(\Lambda^{s}\right)^{*}$.

3. Invariant theory and the Adem relations. In this section we show a connection between the invariant theory of $G L_{2}$ and the Adem relations. Proposition 3.1 is our central observation. It explains why invariant theory should play a role in building resolutions of modules over the Steenrod algebra.

Define an $F_{2}$-linear map $\pi: \Delta_{2} \rightarrow A$ by

$$
\pi\left(v_{1}^{p} v_{2}^{q}\right)=\mathrm{Sq}^{p+1} \mathrm{Sq}^{q+1} .
$$

Here we understand $\mathrm{Sq}^{k}=0$ if $k<0$, and $\mathrm{Sq}^{0}=1$.

Proposition 3.1. $\Gamma_{2} \subset \operatorname{ker} \pi$.

Proof. We use the description of $\Gamma_{2}$ given by (2.9), and will show that $\pi\left(Q_{2,0}^{r} Q_{2,1}^{s}\right)$ $=0$ for all $r \in Z$ and $s \geqslant 0$ by induction on $s$. If $s=0$ we note that $\mathrm{Sq}^{2 r+1} \mathrm{Sq}^{r+1}=0$ in the Steenrod algebra, so the result is proved in this case. Now we carry out the inductive step, using (3.1) to compute for any $\gamma \in \Delta_{2}$.

$$
\pi\left(\frac{Q_{2,1}}{Q_{2,0}} \gamma\right)=\pi\left(\left(\frac{1}{v_{1}}+\frac{1}{v_{2}}\right) \gamma\right)=\alpha \pi(\gamma)
$$

where $\alpha: A \rightarrow A$ is the derivation characterized by $\alpha\left(\mathrm{Sq}^{k}\right)=\mathrm{Sq}^{k-1}$ [4]. So the proposition is proved.

We remark in passing that Proposition 3.1 actually determines the Steenrod algebra. That is, one can use (3.1) to define an $F_{2}$-linear map $\pi$ from $\Delta_{2}$ to the tensor algebra on the formal symbols $\left\{\mathrm{Sq}^{k} \mid k>0\right\}$. The Steenrod algebra results if we impose the relations $\pi\left(\Gamma_{2}\right)=0$. 
4. The chain complexes $\Gamma^{+} M$. Let $M$ be an arbitrary left $A$-module. We will now construct the chain complex $\Gamma^{+} M$ having properties (1.4) and (1.5).

Define an $F_{2}$-linear map $\partial: \Delta_{s} \otimes M \rightarrow \Delta_{s-1} \otimes M$ by

$$
\partial\left(v_{1}^{j_{1}} \cdots v_{s}^{j_{1}} \otimes x\right)=v_{1}^{j_{1}} \cdots v_{s-1}^{j_{1}} \otimes \mathrm{Sq}^{j_{1}+1} x
$$

(we understand $\mathrm{Sq}^{k} x=0$ if $k<0$ ). From (2.20) it is clear that $\partial\left(\Gamma_{s}^{+} \otimes M\right) \subseteq$ $\Gamma_{s-1}^{+} \otimes M$. We define the chain complex $\Gamma^{+} M$ by setting $\left(\Gamma^{+} M\right)_{s}=\Gamma_{s}^{+} \otimes M$ as an $F_{2}$-vector space for all $s \geqslant 0$, and declaring the differential to be the restriction of (4.1) to $\Gamma_{s}^{+} \otimes M$. That the composition

$$
\Gamma_{s}^{+} \otimes M \stackrel{\partial}{\rightarrow} \Gamma_{s-1}^{+} \otimes M \stackrel{\partial}{\rightarrow} \Gamma_{s-2}^{+} \otimes M
$$

is zero follows from Proposition 2.1 (with $q=2$ ) and Proposition 3.1.

We regard the association $M \rightarrow \Gamma^{+} M$ as a functor from $A$-modules to chain complexes over $F_{2}$, and prove at once the following three propositions.

Proposition 4.1. There is a natural isomorphism $H_{0}\left(\Gamma^{+} M\right)=F_{2} \otimes_{A} M$.

Proposition 4.2. If $0 \rightarrow M \rightarrow N \rightarrow P \rightarrow 0$ is a short exact sequence of $A$-modules then $0 \rightarrow \Gamma^{+} M \rightarrow \Gamma^{+} N \rightarrow \Gamma^{+} P \rightarrow 0$ is a short exact sequence of chain complexes.

Proposition 4.3. To any short exact sequence $0 \rightarrow M \rightarrow N \rightarrow P \rightarrow 0$ of $A$-modules is naturally associated a long exact sequence in homology:

$$
\cdots \rightarrow H_{s}\left(\Gamma^{+} N\right) \rightarrow H_{s}\left(\Gamma^{+} P\right) \rightarrow H_{s-1}\left(\Gamma^{+} M\right) \rightarrow \cdots \rightarrow H_{0}\left(\Gamma^{+} P\right) \rightarrow 0 .
$$

Finally we assert

Proposition 4.4. $H_{s}\left(\Gamma^{+} A\right)=0$ if $s>0$.

Proof. Our proof is in fact dual to the argument in $\$ 7.4$ of [1], but we include it to make our paper selfcontained. If $\mathrm{Sq}^{I}=\mathrm{Sq}^{i_{1}} \cdots \mathrm{Sq}^{i_{p}}$ is an "admissible" monomial in $A$, write $l(I)=p$ for its length. Define an increasing filtration on $\Gamma^{+} A$ by setting

$$
F_{p}\left(\Gamma^{+} A\right)_{s}=\Gamma_{s}^{+} \otimes \operatorname{Span}\left\{\mathrm{Sq}^{I} \mid I \text { admissible; } l(I) \leqslant p-s\right\} .
$$

Then $F_{p}\left(\Gamma^{+} A\right)$ is a subcomplex of $\Gamma^{+} A$ for each $p \geqslant 0$. Write $E^{0} \Gamma^{+} A$ for the associated graded object: for each $p \geqslant 0, E_{p}^{0} \Gamma^{+} A=F_{p} \Gamma^{+} A / F_{p-1} \Gamma^{+} A$ is a chain complex. Now, a monomial $y=Q_{s, 0}^{i_{0}} \cdots Q_{s, s-1}^{i_{,}, 1} \otimes \mathrm{Sq}^{k_{1}} \cdots \mathrm{Sq}^{k_{p}}$, in $F_{p}\left(\Gamma^{+} A\right)_{s} / F_{p-1}\left(\Gamma^{+} A\right)_{s}$ will be called admissible if both $\mathrm{Sq}^{k_{1}} \cdots \mathrm{Sq}^{k_{p}}$, is an admissible monomial in $A$, and if $j_{s}>2 k_{1}-2$. Here we are writing $\left(j_{1}, \ldots, j_{s}\right)=$ $\phi\left(i_{0}, \ldots, i_{s-1}\right)$ as in Lemma 2.6; in particular $j_{s}=i_{0}+i_{1}+\cdots+i_{s-1}$. Now define an increasing filtration on $E^{0} \Gamma^{+} A$ by setting

$$
\left(G_{q} E_{0} \Gamma^{+} A\right)_{s}=\left\{\begin{array}{l}
E_{0} \Gamma^{+} A, \quad s<q, \\
\operatorname{Span}\left\{y=Q_{s, 0}^{i_{0}} \cdots Q_{s, s-1}^{i_{s}-1} \otimes \operatorname{Sq}^{I} \mid y \text { admissible }\right\}, \quad s=q, \\
0, \quad s>q,
\end{array}\right.
$$


for all $q \geqslant 0$. Then $G_{q} E_{0} \Gamma^{+} A$ is a subcomplex of $E^{0} \Gamma^{+} A$. Using Proposition 2.4 and Lemma 2.6 we see easily that

$$
H_{s}\left(\frac{G_{q} E_{0} \Gamma^{+} A}{G_{q-1} E_{0} \Gamma^{+} A}\right)= \begin{cases}F_{2} & \text { if } s=q=0, \\ 0 & \text { otherwise. }\end{cases}
$$

Proposition 4.4 follows immediately.

THEOREM 4.5. There is a natural isomorphism $H_{s}\left(\Gamma^{+} M\right) \simeq \operatorname{Tor}_{s}^{A}\left(F_{2}, M\right)$.

Proof. The properties of the functors $M \rightarrow H_{s}\left(\Gamma^{+} M\right)(s=0,1,2, \ldots)$ given by Propositions 4.1, 4.3, and 4.4 are also properties of the functors $M \rightarrow \operatorname{Tor}_{s}^{A}\left(F_{2}, M\right)$; and in fact, the latter functors are characterized by them.

This is Theorem 1.1 of the introduction.

5. Action of the Steenrod algebra on $\left(\Gamma^{+} M\right)_{s}$. Our object in this section is to prove

Theorem 5.1. Let $M$ be a graded A-module. For each fixed $s \geqslant 0,\left(\Gamma^{+} M\right)_{s}$ has a natural left A-action, in such a way that the differential $\mathrm{\partial}:\left(\Gamma^{+} M\right)_{s} \rightarrow\left(\Gamma^{+} M\right)_{s-1}$ is A-linear.

In order to show this, we recall the main results of [8]. There we constructed a functor $R_{+}$from the category of graded $A$-modules to itself. In the present context $\Delta$ seems a more appropriate name for this functor; we will use it here. The main properties of this functor are:

(i) $\Delta M$ is isomorphic to $\Delta_{1} \otimes M$ as a graded $F_{2}$-vector space ( $n o t$ in general as an $A$-module). Here $\Delta_{1}=F_{2}\left[v^{ \pm 1}\right]$ is the algebra of finite Laurent series on a generator $v$ of dimension one, as in (2.12). The $A$-action on $\Delta M$ is given by

$$
\mathrm{Sq}^{a}\left(v^{b} \otimes x\right)=\sum_{j}\left(\begin{array}{c}
b-j \\
a-2 j
\end{array}\right) v^{a+b-j} \otimes \mathrm{Sq}^{j} x
$$

with the binomial coefficient interpreted as in $\S 2$ of [8]. If $g: M \rightarrow N$ is an $A$-linear map, then $\Delta g: \Delta M \rightarrow \Delta N$ is given by $(\Delta g)\left(v^{k} \otimes x\right)=v^{k} \otimes g(x)$.

(ii) If $d: \Delta M \rightarrow M$ is the "evaluation map" given by $d\left(v^{k} \otimes x\right)=\mathrm{Sq}^{k+1} x$, then $d$ is $A$-linear.

These results are contained in Theorem 3.4 of [8].

We can now explain our strategy for proving Theorem 5.1. Write $\Delta_{s} M$ for the result of applying the functor $\Delta$ to $M$ a total of $s$-times. (In [8], $\Delta_{s} M$ was written $R_{+}^{s} M$.) Then as a graded vector space, $\Delta_{s} M$ is obtained by tensoring $M$ with an algebra of finite Laurent series on $s$ generators, each of dimension one. We will identify this algebra of Laurent series with the algebra $\Delta_{s}$ of (2.8) and (2.12), writing

$$
\Delta_{s} M=\Delta_{s} \otimes M=F_{2}\left[v_{1}^{ \pm 1}, \ldots, v_{s}^{ \pm 1}\right] \otimes M
$$

as graded vector spaces. Here the identification of left-hand side with right-hand side is defined inductively by the following rule. If $y \in \Delta_{s-1} M$ has been identified with $v_{1}^{j_{1}} \cdots v_{s-1}^{j_{s-1}} \otimes x$, then $v^{k} \otimes y$ in $\Delta\left(\Delta_{s-1} M\right)=\Delta_{s} M$ is to be identified with $v_{1}^{k} v_{2}^{j_{1}}$ $\cdots v_{s}^{j_{s-1}} \otimes x$ in $\Delta_{s} \otimes M$. (5.2) is an isomorphism of $F_{2}$ vector spaces only. It is not meant to imply anything about $\Delta_{s} M$ as an $A$-module. 
We define $F_{2}$-subspaces $\Delta_{s}^{+} M, \Gamma_{s} M$, and $\Gamma_{s}^{+} M$ of $\Delta_{s} M$, by using the identification (5.2) and writing

$$
\Delta_{s}^{+} M=\Delta_{s}^{+} \otimes M, \quad \Gamma_{s} M=\Gamma_{s} \otimes M, \quad \Gamma_{s}^{+} M=\Gamma_{s}^{+} \otimes M .
$$

We will show

TheOREM 5.2. Let $M$ be a graded $A$-module. Then $\Delta_{s}^{+} M, \Gamma_{s} M$, and $\Gamma_{s}^{+} M$ are all $A$-submodules of $\Delta_{s} M$.

Before stating the proof of Theorem 5.2 we observe that it implies Theorem 5.1. In fact $\left(\Gamma^{+} M\right)_{s}$ was defined in $\S 4$ to be the vector space $\Gamma_{s}^{+} \otimes M$, so Theorem 5.2 gives us a natural $A$-action on $\left(\Gamma^{+} M\right)_{s}$. We demonstrate the $A$-linearity of $\partial:\left(\Gamma^{+} M\right)_{s} \rightarrow$ $\left(\Gamma^{+} M\right)_{s-1}$ by observing that with the identification (5.2), $\partial: \Delta_{s} \otimes M \rightarrow \Delta_{s-1} \otimes M$ of (4.1) is just $\Delta_{s-1} d: \Delta_{s} M \rightarrow \Delta_{s-1} M$, where $d: \Delta M \rightarrow M$ is the $A$-linear evaluation map of (ii) above. So to prove Theorem 5.1, we need only prove Theorem 5.2.

It is helpful to begin with the case in which the $A$-module $M$ is unstable. In this case we define a subobject $R M \subseteq \Delta M$ :

$$
R M=\operatorname{Span}\left\{v^{b} \otimes x \mid b \geqslant \operatorname{dim} x\right\} .
$$

We use (5.1) to check that $R M$ is an $A$-submodule of $\Delta M$, and that $R M$ is unstable if $M$ is. (The functor $R$ was introduced in [10].)

Write $M \otimes N$ for the tensor product of two $A$-modules with diagonal $A$-action. In order to study the $A$-action upon $R M$ we recall another result from [8]: an idea of Haynes Miller.

(iii) Suppose $M$ an unstable $A$-module. Then $f: R M \rightarrow P_{1} \otimes M$ given by

$$
f\left(v^{b} \otimes x\right)=\sum_{j=0}^{b}\left(t^{h-j} \otimes \mathrm{Sq}^{j} x\right)
$$

is an $A$-linear monomorphism.

This comes from $\$ 3$ of [8]. There it was assumed that $M$ was bounded above, but is clear that $f$ can also be defined if $M$ is unstable. An alternate expression for $f$ is (3.3) of [8], valid when $M$ is unstable:

$$
f\left(v^{b} \otimes x\right)=\operatorname{Sq}^{b+1}\left(t^{-1} \otimes x\right) .
$$

The right-hand side of (5.6) refers to the diagonal action of $A$ upon $\Delta_{1} \otimes M$, and the action of $A$ upon $\Delta_{1}$ is as in (2.1) of [8].

Now write $R_{s}$ for the $s$-fold iterate of $R$. Then for any unstable $A$-module $M, R_{s} M$ is naturally an $A$-submodule of $\Delta_{s} M$. For integers $s \geqslant 1$ and unstable $M$, define by induction on $s$ an $A$-linear monomorphism

$$
f_{s}: R_{s} M \rightarrow P_{s} \otimes M
$$

by setting $f_{1}=f$, and declaring commutative the diagram:

$$
\begin{aligned}
& R_{s} M=R R_{s-1} M \stackrel{R f_{s-1}}{\rightarrow} \quad R\left(P_{s-1} \otimes M\right) \\
& f_{s} \downarrow \\
& P_{s} \otimes M \quad \stackrel{\rightarrow}{\cong} P_{1} \otimes\left(P_{s-1} \otimes M\right)
\end{aligned}
$$


(Of course one must be aware that $P_{s-1} \otimes M$ is unstable if $M$ is.) We obtain an equivalent definition of $f_{s}$ by writing $f_{s}=\left(P_{1} \otimes f_{s-1}\right) \circ f_{1}$. As a consequence of this equivalence we have commutativity of the following diagram for all $i, 0 \leqslant i \leqslant s-2$ :

$$
\begin{array}{ccccc}
R^{s} M & \sim & R^{i} R^{2} R^{s-i-2} M & \stackrel{R^{i} f_{2}}{\rightarrow} & R^{i}\left(P_{2} \otimes R^{s-i-2} M\right) \\
f_{s} \downarrow & & & & \downarrow R^{i}\left(P_{2} \otimes f_{s-i-2}\right) \\
P_{s} \otimes M & \stackrel{\rightarrow}{*} & P_{i} \otimes P_{2} \otimes P_{s-i-2} \otimes M & \underset{f_{i}}{\leftarrow} & R^{i}\left(P_{2} \otimes P_{s-i-2} \otimes M\right)
\end{array}
$$

When we make the identification (5.2), elements of the submodule $R_{s} M \subset \Delta_{s} M$ are represented by elements of the submodule $P_{s} \otimes M \subset \Delta_{s} \otimes M$. This observation helps us to understand the following lemma, which gives another property of $f_{s}: R_{s} M \rightarrow P_{s} \otimes M$.

LEMma 5.3. Let $\gamma \otimes x$ represent an element of $R_{s} M$ under the identification (5.2), where $\gamma \in P_{s}$, and $x \in M$ is homogeneous. Then $f_{s}(\gamma \otimes x)=\gamma \otimes x+\sum_{i} \gamma_{i} \otimes x_{i}$ where $\operatorname{dim} x_{i}>\operatorname{dim} x$ for all $x_{i}$ appearing in the sum.

This follows directly from the definition of $f_{s}$.

Our first result en route to Theorem 5.2 is

Lemma 5.4. Let $M$ be an unstable $A$-module. Then the image of $f_{2}: R_{2} M \rightarrow P_{2} \otimes M$ is contained in $P_{2}^{T_{2}} \otimes M$.

Proof. Using (5.5), (5.6), and (5.8) we find

$$
f_{2}\left(v_{1}^{a} v_{2}^{b} \otimes x\right)=\mathrm{Sq}^{a+1} \sum_{j=0}^{b} t_{1}^{-1} t_{2}^{b-j} \otimes \mathrm{Sq}^{j} x
$$

But since $v_{1}^{a} v_{2}^{b} \otimes x$ lies in $R_{2} M$ we have $a \geqslant b+\operatorname{dim} x$. Then it follows from the instability of $P_{2} \otimes M$ that the right-hand side of (5.10) is unaffected by the substitution $t_{1} \rightarrow t_{1}, t_{2} \rightarrow t_{1}+t_{2}$. This completes the proof.

Lemma 5.5. Let $M$ be unstable. Then $f_{2}$ carries $R_{2} M \cap \Gamma_{2} M$ into $P_{2}^{G L_{2}} \otimes M$.

Proof. $R_{2} M \cap \Gamma_{2} M$ is spanned by elements of the form $\gamma \otimes x$, where $x \in M$ and $\gamma=\sum_{i} v_{1}^{a_{i}} v_{2}^{b_{i}}$ lies in $\Gamma_{2}$. Then by Proposition 3.1, $\sum_{i} \mathrm{Sq}^{a_{i}+1} \mathrm{Sq}^{b_{i}+1}=0$. Then in $P_{2} \otimes M$ we have

$$
\begin{aligned}
0= & \sum_{i} \mathrm{Sq}^{a_{i}+1} \mathrm{Sq}^{b_{i}+1}\left(t_{1}^{-1} t_{2}^{-1} \otimes x\right) \\
= & \sum_{i} \mathrm{Sq}^{a_{i}+1}\left(t_{1}^{-1} \mathrm{Sq}^{b_{i}+1}\left(t_{2}^{-1} \otimes x\right)\right) \\
& +\sum_{i} \mathrm{Sq}^{a_{i}+1} \sum_{j=0}^{b_{i}} t_{1}^{b_{i}-j} \mathrm{Sq}^{j}\left(t_{2}^{-1} \otimes x\right) .
\end{aligned}
$$

Using (5.6) we see that (5.11) implies

$$
f_{2}(\gamma \otimes x)=\sum_{i} \mathrm{Sq}^{a_{i}+1}\left(t_{1}^{-1} \mathrm{Sq}^{b_{i}+1}\left(t_{2}^{-1} \otimes x\right)\right)=\sum_{i} \mathrm{Sq}^{a_{i}+1} \sum_{j=0}^{b_{i}} t_{1}^{b_{i}-j} \operatorname{Sq}^{j}\left(t_{2}^{-1} \otimes x\right) \text {. }
$$


But if $\gamma \otimes x$ lies in $R_{2} M$ then $a_{i} \geqslant b_{i}+\operatorname{dim} x$ for all $i$, so using the instability of $P_{2} \otimes M$ we obtain from (5.12),

$$
f_{2}(\gamma \otimes x)=\sum_{i} \mathrm{Sq}^{a_{i}+1} \sum_{j=0}^{b_{1}} t_{1}^{b_{i}-j} t_{2}^{-1} \otimes \mathrm{Sq}^{j} x .
$$

Comparing this expression for $f_{2}(\gamma \otimes x)$ with the one coming from (5.10), we find that $f_{2}(\gamma \otimes x)$ is unchanged by the substitution $t_{1} \rightarrow t_{2}, t_{2} \rightarrow t_{1}$. Appeal to Lemma 5.4 completes the proof.

Now we generalize Lemma 5.5.

Lemma 5.6. Let $M$ be unstable. Then $f_{s}$ carries $R_{s} M \cap \Gamma_{s} M$ into $P_{s}^{G I_{s}} \otimes M$.

Proof. Let $i$ be any integer, $0 \leqslant i \leqslant s-2$. As vector spaces we can identify $\Delta_{s} M \cong \Delta_{s} \otimes M \cong \Delta_{i} \otimes \Delta_{2} \otimes \Delta_{s-i-2} \otimes M$, where we are using the maps $\psi_{p . q}$ of (2.13) to define the isomorphism

$$
\Delta_{s} \stackrel{\psi_{1, s-i}}{\rightarrow} \Delta_{i} \otimes \Delta_{s-i} \stackrel{\lambda_{1} \otimes \psi_{2 . s}}{\rightarrow} \cdot{ }^{\prime} \Delta_{i} \otimes \Delta_{2} \otimes \Delta_{s-i-2} .
$$

Then it follows from Proposition 2.1 that any $y \in \Gamma_{s} M$ can be written as a sum of elements of the form $\gamma_{1} \otimes \gamma_{2} \otimes \gamma_{3} \otimes x$, with $\gamma_{1} \in \Delta_{i}, \gamma_{2} \in \Gamma_{2}, \gamma_{3} \in \Delta_{s-i-2}$. Then it follows from (5.9) and Lemma 5.5 that $f_{s}(y)$ lies in $P_{i} \otimes P_{2}^{G L_{2}} \otimes P_{s-i-2} \otimes M$. But this is true for any $i$ with $0 \leqslant i \leqslant s-2$ so our lemma is proved.

Lemma 5.6 can be used to prove its own converse.

Lemma 5.7. Let $M$ be unstable. Then $f_{s}^{-1}\left(P_{s}^{G L_{s}} \otimes M\right) \subseteq R_{s} M \cap \Gamma_{s} M$.

Proof. This follows easily from Lemmas 5.3 and 5.6.

We now can prove a special case of Theorem 5.2.

Proposition 5.8. Let $M$ be unstable. Then $R_{s} M \cap \Gamma_{s} M$ is an A-submodule of $R_{s} M$.

Proof. This follows at once from Lemmas 5.6 and 5.7, and the obvious fact that $P_{s}^{G L_{s}} \otimes M$ is an $A$-submodule of $P_{s} \otimes M$.

We next aim to generalize Proposition 5.8, by showing that if $M$ is unstable then $\Gamma_{s} M$ is an $A$-submodule of $\Delta_{s} M$. To this end we continue to recall from [8] the properties of the functor $\Delta$.

(iv) The natural pairing $\xi=\xi(M, N): \Delta M \otimes \Delta N \rightarrow \Delta(M \otimes N)$ given by

$$
\xi\left(\left(v^{a} \otimes x\right) \otimes\left(v^{b} \otimes y\right)\right)=v^{a+b} \otimes(x \otimes y)
$$

is an $A$-linear map. In particular if $M$ is an $A$-algebra with product $\mu: M \otimes M \rightarrow M$ (as in $[8, \S 2]$ ), then $\Delta M$ is also an $A$-algebra if we define the product by

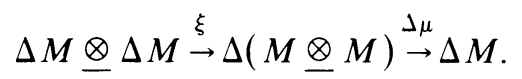

(v) If $M=F_{2}$, the identification (5.2) of $\Delta_{s} F_{2}$ with $\Delta_{s}$ is an isomorphism of $A$-algebras.

Property (iv) above is Proposition 7.1 of [8]. (v) is obtained by combining Propositions 9.1 and 9.2 of [8]. Of course, the $A$-action on $\Delta_{s}$ that we refer to here is obtained by applying the process of localizing an $A$-algebra, due to Wilkerson 
[11, Proposition 2.1], to the $A$-algebra $P_{s}^{T_{v}}$. Alternately, one can start with the action of $A$ upon $P_{s}$ that comes from identifying $P_{s}$ with the cohomology of a product of infinite projective spaces, pass by Wilkerson's procedure to an action of $A$ upon the localization $\Phi_{s}=\left(P_{s}\right)_{S(s)}$, and then restrict this action to $\Delta_{s}$. These matters are all explained in $\S \S 2,8$ and 9 of [8].

For any $A$-module $M$, define an action

$$
\Delta_{s} \otimes \Delta_{s} M \rightarrow \Delta_{s} M
$$

by using the identification (5.2) and writing $\gamma\left(\gamma^{\prime} \otimes x\right)=\gamma \gamma^{\prime} \otimes x$.

Proposition 5.9. With diagonal A-action as indicated on the left-hand side, (5.15) is an A-linear map.

Proof. (5.15) can be obtained by repeated use of the pairing (5.14)

Now we can extend Proposition 5.8.

Proposition 5.10. Let $M$ be unstable. Then $\Gamma_{s} M$ is an $A$-submodule of $\Delta_{s} M$.

Proor. Suppose we wish to show that $\mathrm{Sq}^{h}(\gamma \otimes x)$ lies in $\Gamma_{s} M$, where $\gamma \in \Gamma_{s}$ and we are using (5.2) to label elements of $\Delta_{s} M$. But it is easy to see that we can choose elements $\alpha, \beta \in \Gamma_{s}$ such that $\gamma=\alpha \beta$ and $\beta \otimes x$ lies in $R_{s} M \cap \Gamma_{s} M$. Then by Proposition 5.9 we have

$$
\mathrm{Sq}^{h}(\gamma \otimes x)=\sum_{i+j, h} \mathrm{Sq}^{\prime} \alpha \cdot \mathrm{Sq}^{\prime}(\beta \otimes x)
$$

By Proposition 5.8, each $\mathrm{Sq}^{\prime}(\beta \otimes x)$ lies in $\Gamma_{s} M$. Also, reference to the invarianttheoretic definitions of $\Gamma_{s}$ and $\Delta_{s}$ shows that $\Gamma_{s}$ is an $A$-subalgebra of $\Delta_{s}$, so each $\mathrm{Sq}^{i} \alpha$ lies in $\Gamma_{s}$. Hence the right-hand side of (5.16) lies in $\Gamma_{s} M$ and we are done.

We can now easily remove from Proposition 5.10 the assumption that $M$ is unstable. First we have

Proposition 5.11. Let $M$ be a graded $A$-module, bounded above. Then $\Gamma_{s} M$ is an $A$-submodule of $\Delta_{s} M$.

Proof. Suppose we wish to show that $\mathrm{Sq}^{k}(\gamma \otimes x)$ lies in $\Gamma_{s} M$, where $\gamma \in \Gamma_{s}$. Let $M^{\prime} \subseteq M$ be the $A$-submodule consisting of all elements $y$ for which $\operatorname{dim} x \leqslant \operatorname{dim} y$. It is enough to show that $\mathrm{Sq}^{k}(\gamma \otimes x)$ lies in $\Gamma_{s} M^{\prime}$. But the $r$-fold suspension $\Sigma^{r} M^{\prime}$ is unstable for $r$ sufficiently large ( $M$ is bounded above) so our result follows from the fact that the functors $\Sigma$ and $\Delta$ commute.

Proposition 5.12. Let $M$ be an arbitrary graded $A$-module. Then $\Gamma_{s} M$ is an A-submodule of $\Delta_{s} M$.

Proof. This follows easily from the case in which $M$ is bounded above.

We continue toward a proof of Theorem 5.2 by showing

Proposition 5.13. Let $M$ be a graded A-module. Then $\Delta_{s}^{+} M$ is an A-submodule of $\Delta_{s} M$.

Proof. By Proposition 2.3, it will suffice to show that if the monomial $v_{1}^{j_{1}} \cdots v_{s}^{j_{s}}$ lies in $\Delta_{s}^{+}$, then for any $x \in M$ the element $\operatorname{Sq}^{k}\left(v_{1}^{j_{1}} \cdots v_{s}^{j_{s}} \otimes x\right)$ lies in $\Delta_{s}^{+} M$. But 
repeated use of (5.1) shows that $\mathrm{Sq}^{k}\left(v_{1}^{j_{1}} \cdots v_{s}^{j,} \otimes x\right)$ can be written as a sum of terms of the form $v_{1}^{l_{1}} \cdots v_{s}^{l_{s}} \otimes y$, where

$$
j_{1} \leqslant l_{1}, \ldots, j_{s} \leqslant l_{s} .
$$

Now each $v_{i}$ lies in $\Delta_{s}^{+}$(apparent from (2.2), (2.10) and (2.15)), and since $\Delta_{s}^{+}$is a subalgebra of $\Delta_{s}$, it follows from (5.17) that $v_{1}^{l_{1}} \cdots v_{s}^{l_{s}}$ lies in $\Delta_{s}^{+}$. The proof is complete.

Proposition 5.14. Let $M$ be a graded A-module. Then $\Gamma_{s}^{+} M$ is an A-submodule of $\Delta_{s} M$.

Proof. Since $\Gamma_{s}^{+}=\Gamma_{s} \cap \Delta_{s}^{+}$, our result follows from Propositions 5.12 and 5.13.

Combining Propositions 5.12-5.14, we find that we have proved Theorem 5.2. As we remarked after the statement of that theorem, Theorem 5.1 follows immediately.

Since we have now shown that the chain complex $\Gamma^{+} M$ has a natural $A$-action in each homological degree, it is natural to ask about the induced $A$-action on each $\operatorname{Tor}_{s}^{A}\left(F_{2}, M\right)=H_{s}\left(\Gamma^{+} M\right)$.

TheOREM 5.15. The action of $A$ upon $\Gamma^{+} M$ induces the trivial action of $A$ upon $\operatorname{Tor}_{s}^{A}\left(F_{2}, M\right)$, for each $s \geqslant 0$.

Proof. Induct on $s$. For the case $s=0$ observe that the chain complex $\Gamma^{+} M$ in homological dimensions 0 and 1 looks like

$$
\operatorname{Span}\left\{v_{1}^{k} \mid k \geqslant 0\right\} \otimes M \stackrel{\partial}{\rightarrow} M
$$

where $\partial\left(v_{1}^{k} \otimes x\right)=\mathrm{Sq}^{k+1} x$. The cases $s>0$ do not follow in such a straightforward way from the structure of $\Gamma^{+} M$. But we use the inductive assumption: suppose the result proved for the case $s-1$, for some $s \geqslant 1$ and all $A$-modules $M$. Let $\pi: F \rightarrow M$ be a mapping from a free $A$-module onto $M$, and let $K$ be the kernel. Then $0 \rightarrow \Gamma^{+} K \rightarrow \Gamma^{+} F \rightarrow \Gamma^{+} M \rightarrow 0$ is a short exact sequence of chain complexes of $A$-modules, of which the middle complex is acyclic in positive homological dimensions. So the triviality of the $A$-action on $H_{s}\left(\Gamma^{+} M\right)$ follows from the triviality of the $A$-action on $H_{s-1}\left(\Gamma^{+} K\right)$.

Theorems 5.1 and 5.15 together give Theorem 1.3 of the introduction.

6. An extension of the lambda algebra. The next three sections are devoted to comparing the chain complex $\Gamma^{+} F_{2}$ with the dual of the lambda algebra. We will give $\Gamma^{+} F_{2}$ the structure of a differential coalgebra, and prove Theorem 1.2. We begin by defining lambảa by embedding it in a somewhat larger differential algebra, which we will call "theta". This algebra was described to me by Bousfield and by Priddy, and was known to the authors of [1].

Let $L$ be the graded $F_{2}$-vector space with basis consisting of the symbols $\left\{\lambda_{k} \mid k\right.$ integral, $k \geqslant-1\}$, where $\operatorname{deg} \lambda_{k}=k$. Let Tens $L$ be the free associative algebra generated by $L$. Then Tens $L$ is a bigraded algebra if we write bideg $\lambda_{k}=(1, k)$. We will usually supress the second of these two degrees ("interval degree") and record 
only the first ("homological degree"). In (Tens $L)^{2}=L \otimes L$ we define a family of homogeneous elements

$$
\lambda(p, q)=\sum_{j \geqslant 0}\left(\begin{array}{l}
p \\
j
\end{array}\right) \lambda_{2 q+j-1} \lambda_{p+q-j-1} \quad(p, q \geqslant 0)
$$

and then define $\Theta$ to be the bigraded algebra obtained from Tens $L$ by imposing the relations $\lambda(p, q)=0(p, q \geqslant 0)$. These relations are of two kinds. Those involving $\lambda_{-1}$ are precisely the statements $\lambda(p, 0)=0$ :

$$
\begin{gathered}
\lambda_{-1} \lambda_{-1}=0 \\
\lambda_{-1} \lambda_{p-1}+\sum_{j=1}^{p-1}\left(\begin{array}{l}
p \\
j
\end{array}\right) \lambda_{j-1} \lambda_{p-j-1}+\lambda_{p-1} \lambda_{-1}=0 \quad(p>0)
\end{gathered}
$$

but only the generators $\left\{\lambda_{k} \mid k \geqslant 0\right\}$ appear in $\lambda(p, q)$ if $q>0$.

Now we let $\Lambda$ be the subalgebra of $\Theta$ that is generated by the symbols $\left\{\lambda_{k} \mid k \geqslant 0\right\}$. Then $\Lambda$ is defined by the relations $\lambda(p, q)=0$ for which $p \geqslant 0$ and $q>0$. This definition is the same as that originally given in [1], but observe that we are writing the product in the order opposite to that used in [1]. We note, as in [2, p. 459], that for each $s \geqslant 1$, a basis for $\Lambda^{s}$ is given by the set of "admissibles":

$$
\left\{\lambda_{j_{1}} \lambda_{j_{2}} \cdots \lambda_{j_{s}} \mid 0 \leqslant j_{1}, j_{1} \leqslant 2 j_{2}, \ldots, j_{s-1} \leqslant 2 j_{s}\right\}
$$

while $\Lambda^{0}$ is spanned by the unit.

Define a homomorphism $d: \Theta \rightarrow \Theta$ by

$$
d x=\lambda_{-1} x+x \lambda_{-1} \text {. }
$$

This definition was shown me by Bousfield and Priddy. The map $d$ is clearly a derivation, and since $\lambda_{-1} \lambda_{-1}=0$ we have $d d=0$. (6.2) also implies that

$$
d \lambda_{p-1}=\sum_{j=1}^{p-1}\left(\begin{array}{l}
p \\
j
\end{array}\right) \lambda_{j-1} \lambda_{p-j-1} \quad(p>0)
$$

so that $\Lambda$ is a subdifferential algebra of $\Theta$. This completes our description of the differential algebras $\Theta$ and $\Lambda$.

We close this section by defining a certain derivation $\chi: \Theta \rightarrow \Theta$; it will be useful in $\S 7$ when we compare $\Gamma^{+}$to $\Theta^{*}$. In fact there is a unique derivation $\chi$ : Tens $L \rightarrow$ Tens $L$ that satisfies

$$
\chi\left(\lambda_{k}\right)=\lambda_{k+1} \quad(k \geqslant-1) .
$$

An easy computation shows

$$
\chi \lambda(p, q)=\lambda(p+1, q)
$$

so $\chi$ passes to a derivation on $\Theta$ satisfying (6.6).

7. Invariants of $G L_{s}$ and the dual of $\Theta$. Our object in this section is to construct a map of coalgebras $k: \Gamma \rightarrow \Theta^{*}$.

If $V$ is a graded (or bigraded) vector space, write $V^{*}$ for its graded (bigraded) dual: $\left(V^{*}\right)_{m}=\left(V_{m}\right)^{*}$ (or $\left.\left(V^{*}\right)_{p, q}=\left(V_{p, q}\right)^{*}\right)$. Then the equations $\left(V^{*}\right)^{*}=V$ and $(V \otimes W)^{*}=V^{*} \otimes W^{*}$ are valid as long as $V$ and $W$ are finite dimensional in each 
degree (or bidegree). In particular, we may regard (Tens $L)^{*}, \Theta^{*}$, and $\Lambda^{*}$ as coalgebras.

Define the degree-preserving $F_{2}$-linear $k_{s}: \Delta_{s} \rightarrow\left(L^{\otimes s}\right)^{*}$ by

$$
\left\langle k_{s}\left(v_{1}^{i_{1}} \cdots v_{s}^{i_{s}}\right), \lambda_{j_{1}} \otimes \cdots \otimes \lambda_{j_{s}}\right\rangle=\delta_{i_{1}, j_{1}} \cdots \delta_{i_{s}, j_{s}}
$$

for each $s \geqslant 1$, where $\delta_{i, j}$ is the Kronecker delta. Let $k_{0}: \Delta_{0} \rightarrow\left(L^{\otimes 0}\right)^{*}$ be the identity on $F_{2}$. With $\psi_{p, q}: \Delta_{s} \rightarrow \Delta_{p} \otimes \Delta_{q}$ as in (2.13) we have clearly

$$
\left\langle k_{s}(\gamma), \alpha \beta\right\rangle=\left\langle\left(k_{p} \otimes k_{q}\right) \psi_{p, q}(\gamma), \alpha \otimes \beta\right\rangle
$$

for $\alpha \in L^{\otimes p}, \beta \in L^{\otimes q}, \gamma \in \Delta_{s}$. (We are identifying $\left(L^{\otimes \mathrm{p}} \otimes L^{\otimes q}\right)^{*}$ with $\left(L^{\otimes p}\right)^{*} \otimes$ $\left(L^{\otimes q}\right)^{*}$ in (7.2).) That is, if the maps $k_{s}$ for $s \geqslant 0$ are assembled to form $k: \Delta \rightarrow(\text { Tens } L)^{*}$ then

Proposition 7.1. $k: \Delta \rightarrow(\text { Tens } L)^{*}$ is a morphism of graded coalgebras.

We now aim to show that the restriction $k_{s} \mid \Gamma_{s}$ is actually a map from $\Gamma_{s}$ to $\left(\Theta^{s}\right)^{*}$; that is, if $\gamma$ lies in $\Gamma_{s}$ then the functional $k_{s}(\gamma)$ annihilates the relations (6.1) by which $\Theta$ is defined. We begin by proving this in the case $s=2$.

LEMMA 7.2. For any $\gamma \in \Gamma_{2}$ and any integers $p, q \geqslant 0$ we have

$$
\left\langle k_{2}(\gamma), \lambda(p, q)\right\rangle=0 \text {. }
$$

Proof. It suffices to prove this for the case $\gamma=Q_{2,0}^{a} Q_{2,1}^{b}$, where $a, b$ are integers and $0 \leqslant b$. In the case $b=0$ we note that the relation $\left\langle k_{2}\left(Q_{2.0}^{a}\right), \lambda(p, q)\right\rangle=0$ follows easily from $(6.1),(7.1)$, and the fact that $\left(\begin{array}{c}3 l+1 \\ 2 l+1\end{array}\right)=0$ modulo 2 for all values of the integer $l$. Now we induct on $b$. Suppose (7.3) proved for $\gamma=Q_{2.0}^{a} Q_{2.1}^{b}$ for some fixed $b \geqslant 0$ and all values of $a, p$, and $q$. Define $\rho: \Delta_{2} \rightarrow \Delta_{2}$ by writing

$$
\rho(\delta)=\frac{Q_{2,1}}{Q_{2,0}} \delta=\left(\frac{1}{v_{1}}+\frac{1}{v_{2}}\right) \delta
$$

for each $\delta \in \Delta_{2}$. Clearly the diagram

$$
\begin{array}{ccc}
\Delta_{2} & \stackrel{k_{2}}{\rightarrow} & (L \otimes L)^{*} \\
\rho \downarrow & & \downarrow \chi^{*} \\
\Delta_{2} & \underset{k_{2}}{\rightarrow} & (L \otimes L)^{*}
\end{array}
$$

commutes, where $\chi$ is the derivation of Tens $L$ defined in the previous section. Now if we let $\gamma=Q_{2,0}^{a} Q_{2,1}^{b}$ we have

$$
\begin{aligned}
\left\langle k_{2}\left(Q_{2,0}^{a-1} Q_{2,1}^{b+1}\right), \lambda(p, q)\right\rangle & =\left\langle k_{2} \rho(\gamma), \lambda(p, q)\right\rangle=\left\langle\chi^{*} k_{2}(\gamma), \lambda(p, q)\right\rangle \\
& =\left\langle k_{2}(\gamma), \chi \lambda(p, q)\right\rangle=\left\langle k_{2}(\gamma), \lambda(p+1, q)\right\rangle=0
\end{aligned}
$$

where we have used (6.7) and the inductive assumption. So our proof of (7.3) is complete.

Now Lemma 7.2 generalizes to

Proposition 7.3. Let $\beta \in L^{\otimes s}$ lie in the two-sided ideal of Tens $L$ that is generated by the elements $\lambda(p, q)$. Then $\left\langle k_{s}(\gamma), \beta\right\rangle=0$ for any $\gamma \in \Gamma_{s}$. 
Proof. We may assume $\beta=\alpha_{1} \alpha_{2} \alpha_{3}$ where $\alpha_{1} \in L^{\otimes r}, \alpha_{2}=\lambda(p, q) \in L^{\otimes 2}$, and $\alpha_{3} \in L^{\otimes s-r-2}$ for some $r$. Our result then follows from (7.2), Proposition 2.1, and Lemma 7.2.

It follows from Proposition 7.3 that the restriction of $k_{s}: \Delta_{s} \rightarrow\left(L^{\otimes s}\right)^{*}$ to $\Gamma_{s}$ gives an $F_{2}$-linear map

$$
k_{s}: \Gamma_{s} \rightarrow\left(\Theta^{s}\right)^{*} \text {. }
$$

If we assemble the various $k_{s}(s \geqslant 0)$ to form $k: \Gamma \rightarrow \Theta^{*}$, then from Propositions 2.1 and 7.1 we have

Proposition 7.4. $k: \Gamma \rightarrow \Theta^{*}$ is a morphism of graded coalgebras.

8. Invariants of $G L_{s}$ and the dual of $\Lambda$. In this section we will use the coalgebra map $k: \Gamma \rightarrow \Theta^{*}$ to show how $\Gamma^{+}$can be regarded as a quotient coalgebra of $\Gamma$. We will also prove the isomorphism of differential coalgebras $\left(\Gamma^{+}=\Gamma^{+} F_{2}\right) \rightarrow \Lambda^{*}$ that we promised in Theorem 1.2.

Let $\Gamma_{s}^{+} \subseteq \Gamma_{s}$ be the subalgebra defined by (2.17). Restricting $k_{s}$ of (7.6) to this subalgebra and then composing with the natural projection $\left(\Theta^{s}\right)^{*} \rightarrow\left(\Lambda^{s}\right)^{*}$ we get an $F_{2}$-linear map

$$
l_{s}: \Gamma_{s}^{+} \rightarrow\left(\Lambda^{s}\right)^{*} \text {. }
$$

PROPOSITION 8.1. $l_{s}$ is an isomorphism for each $s \geqslant 0$.

Proof. Let us take as a basis for $\left(\Lambda^{s}\right)^{*}$ the one dual to the basis of admissibles (6.3), writing a typical element $\left(\lambda_{j_{1}} \cdots \lambda_{j_{s}}\right)^{*}$, where $\left(j_{1}, \ldots, j_{s}\right)$ lies in the set $J_{s}$ defined by (2.23). We order this basis antilexicographically. Now take as a basis for $\Gamma_{s}^{+}$the set of monomials given in Proposition 2.4. This basis is labeled by the sequences $\left(i_{0}, \ldots, i_{s-1}\right)$ which lie in the set $I_{s}$, defined by (2.22). But from (7.1) and Lemma 2.7 we find that

$$
l_{s}\left(Q_{s, 0}^{i_{0}} \cdots Q_{s, s-1}^{i_{s-1}}\right)=\left(\lambda_{j_{1}} \cdots \lambda_{j_{s}}\right) *+\text { smaller basis elements }
$$

where $\left(i_{0}, \ldots, i_{s-1}\right)$ is any element of $I_{s}$, and $\phi\left(i_{0}, \ldots, i_{s-1}\right)=\left(j_{1}, \ldots, j_{s}\right)$. Our result now follows from Lemma 2.6.

By identifying $\Gamma_{s}^{+}$with $\left(\Gamma^{+} F_{2}\right)_{s}$ we obtain a differential $\partial: \Gamma_{s}^{+} \rightarrow \Gamma_{s-1}^{+}$defined as in (4.1). Clearly this differential is obtained by restricting to $\Gamma_{s}^{+}$the map ว: $\Delta_{s} \rightarrow \Delta_{s-1}$ given by

$$
\partial\left(v_{1}^{j_{1}} \cdots v_{s}^{j_{s}}\right)= \begin{cases}v_{1}^{j_{1}} \cdots v_{s-1}^{j_{s-1}} & \text { if } j_{s}=-1 \\ 0 & \text { if } j_{s} \neq-1\end{cases}
$$

On the other hand the differential $d . \Lambda^{s-1} \rightarrow \Lambda^{s}$, defined either by (6.4) or (6.5), dualizes to $d^{*}:\left(\Lambda^{s}\right)^{*} \rightarrow\left(\Lambda^{s-1}\right)^{*}$, and we augment Proposition 8.1 by proving

Proposition 8.2. For each $s \geqslant 1$ the following diagram commutes:

$$
\begin{array}{lll}
\Gamma_{s}^{+} & \stackrel{l_{s}}{\rightarrow} & \left(\Lambda^{s}\right)^{*} \\
\partial \downarrow & & \downarrow d^{*} \\
\Gamma_{s-1}^{+} & \stackrel{I}{s-1}^{\rightarrow} & \left(\Lambda^{s-1}\right)^{*}
\end{array}
$$


Proof. Let $\gamma \in \Gamma_{s}^{+}$be given, and consider the expansion (2.21). We will show

$$
l_{s-1} \partial\left(v_{1}^{j_{1}} \cdots v_{s}^{j_{s}}\right)=d^{*} l_{s}\left(v_{1}^{j_{1}} \cdots v_{s}^{j_{1}}\right)
$$

for each monomial on the right of (2.21). Consider first the case $j_{s}=-1$. Evaluating both sides of (8.4) on a test element of $\Lambda^{s-1}$ we find with the aid of (7.1),

$$
\left\langle l_{s, 1} \partial\left(v_{1}^{j} \cdots v_{s}^{-1}\right), \lambda_{k_{1}} \cdots \lambda_{k_{1}, 1}\right\rangle=\delta_{j_{1}, k_{1}} \cdots \delta_{j_{1}, k, k_{1}}
$$

and with the aid of (6.4),

$$
\begin{aligned}
\left\langle d^{*} l_{s}\left(v_{1}^{j_{1}} \cdots v_{s}^{-1}\right), \lambda_{k_{1}} \cdots \lambda_{k_{,}, 1}\right\rangle= & \left\langle l_{s}\left(v_{1}^{j_{1}} \cdots v_{s}^{-1}\right), \lambda_{-1} \lambda_{k_{1}} \cdots \lambda_{k_{,}, 1}\right\rangle \\
& +\left\langle l_{s}\left(v_{1}^{j_{1}} \cdots v_{s}^{-1}\right), \lambda_{k_{1}} \cdots \lambda_{k_{,-1}} \lambda_{-1}\right\rangle .
\end{aligned}
$$

But Lemma 2.5 shows that the first term on the right of (8.6) is zero. So the right-hand sides of (8.5) and (8.6) agree, and (8.4) is proved in the case $j_{s}=-1$. If $j_{s} \neq-1$ the proof is similar; both sides of (8.4) are zero. So the proposition is proved.

We now assemble the maps $l_{s}(s \geqslant 0)$ to obtain $l: \Gamma^{+} F_{2} \rightarrow \Lambda^{*}$. Combining Propositions 8.1 and 8.2 we have

Proposition 8.3. l: $\Gamma^{+} F_{2} \rightarrow \Lambda^{*}$ is an isomorphism of chain complexes.

We now wish to give $\Gamma^{+} F_{2}$ the structure of a differential coalgebra, and to prove $l$ an isomorphism of differential coalgebras. We observed in $\S 2$ that $\Gamma^{+} F_{2} \equiv \Gamma^{+}$is not a subcoalgebra of $\Gamma$. Nevertheless, it is a quotient coalgebra of $\Gamma$. We show this in the following way.

Consider the coalgebra morphism $k: \Gamma \rightarrow \Theta^{*}$ constructed in $§ 7$. Composing $k$ with the projection $\Theta^{*} \rightarrow \Lambda^{*}$ we obtain a coalgebra morphism $\bar{k}: \Gamma \rightarrow \Lambda^{*}$. But the restriction of $\bar{k}$ to $\Gamma^{+}$is the isomorphism $l: \Gamma^{+} \rightarrow \Lambda^{*}$. Hence if we define $\Gamma^{-}$to be the vector space kernel

$$
\Gamma^{-}=\operatorname{ker}\left(\bar{k}: \Gamma \rightarrow \Lambda^{*}\right)
$$

we get an internal direct sum decomposition:

$$
\Gamma=\Gamma^{+} \oplus \Gamma^{-} .
$$

On the other hand, $\bar{k}$ is a map of coalgebras, so $\Gamma^{-}$is a two-sided coideal of $\Gamma$. Hence, by identifying $\Gamma^{+}$with the quotient $\Gamma / \Gamma^{-}$we obtain a coalgebra structure on $\Gamma^{+}$, and since $\bar{k}$ is a coalgebra morphism, so is $l: \Gamma^{+} \rightarrow \Lambda^{*}$. Finally we observe that $\Lambda^{*}$ is a differential coalgebra, and since $l$ is an isomorphism that preserves differential and coproduct, then $\Gamma^{+}$must also be a differential coalgebra. We summarize:

THEOREM 8.4. $l: \Gamma^{+} \rightarrow \Lambda^{*}$ is an isomorphism of differential coalgebras.

This is Theorem 1.2 of the Introduction.

Although there is a nice formula that determines the coproduct on $\Gamma$ (Madsen's formula (2.14) above), there seems to be no equally simple description of the coproduct on $\Gamma^{+}$. Although Madsen's formula is still valid in $\Gamma^{+}$, we can no longer claim that by itself it determines the coproduct. This is because when we identify $\Gamma_{s}^{+}$ with $\Gamma_{s} / \Gamma_{s}^{-}$there is no longer any algebra structure on $\Gamma_{s}^{+}$, and there is no sense in which the elements $Q_{s, i}$ can be regarded as generators. 
Fortunately, it is not hard to compute the coproduct in $\Gamma^{+}$on any particular element one may choose. This is because a description of $\Gamma^{-}$is available that is more explicit than (8.7), enabling one to compute the direct sum decomposition (8.8) in any special case. In fact, let $\Delta_{s}^{-}$be the $F_{2}$-subspace of $\Delta_{s}$ defined by

$$
\Delta_{s}^{-}=\operatorname{Span}\left\{v_{1}^{i_{1}} \cdots v_{s}^{i_{1}} \mid i_{j}<0 \text { for at least one } j\right\} .
$$

Then it follows easily from (7.1) and (8.7) that

$$
\Gamma_{s}^{-}=\Gamma_{s} \cap \Delta_{s}^{-} \text {. }
$$

Notice that the fact that $\Gamma^{-}$is a coideal of $\Gamma$ follows directly from (8.9). In fact it is clear from (2.13) that $\Delta^{-}=\bigoplus_{s \geqslant 0}\left(\Delta_{s}^{-}\right)$is a coideal of $\Delta$.

9. Residue theory. In this section we study a little further the differential $\partial: \Gamma_{s}^{+} \rightarrow \Gamma_{s-1}^{+}$that comes from identifying $\Gamma_{s}^{+}$and $\Gamma_{s-1}^{+}$with the chain groups $\left(\Gamma^{+} F_{2}\right)_{s}$ and $\left(\Gamma^{+} F_{2}\right)_{s-1}$, respectively. This differential is the restriction of the map $\partial: \Delta_{s} \rightarrow \Delta_{s-1}$ defined by (8.2), and we are going to show that (8.2) is a kind of "residue". Roughly speaking, to compute $\partial \gamma$, one regards $\gamma$ as a rational function in $t_{1}, t_{2}, \ldots, t_{s}$, and expands $\gamma$ in a Laurent series about the origin of the $t_{s}$-plane. Then $\partial \gamma$ is the coefficient of $t_{s}^{-1}$.

We proceed to make this idea precise. Let $R$ be any commutative ring with unit; and $t$ an indeterminate. Start with the power series ring $R[[t]]$ and invert the elements of the multiplicative subset $\left\{1, t, t^{2}, \ldots\right\}$. Denote the resulting localization by $R[[t]]^{\prime}$. Then a typical element of $R[[t]]^{\prime}$ is a formal sum $\sum_{-\infty}^{\infty} r_{k} t^{k}$ in which $r_{k}=0$ for all $k$ sufficiently negative. The key property of $R[[t]]^{\prime}$ is given by

LemMa 9.1. Let $f(t)=\sum_{k=0}^{n} a_{k} t^{k}$ be a nonzero element in the polynomial ring $R[t]$. Let $k_{0}$ be the least integer for which $a_{k_{0}} \neq 0$. If $a_{k_{0}}$ is invertible in $R$, then $f(t)$ is invertible in $R[[t]]^{\prime}$.

This is well known.

We define a homomorphism of $R$ modules res: $R[[t]]^{\prime} \rightarrow R$ by

$$
\operatorname{res} \sum_{-\infty}^{\infty} r_{k} t^{k}=r_{-1} \text {. }
$$

We can use this construction to define a residue map from $\Delta_{s}$ to $\Delta_{s-1}$. Observe that $V_{s}$ can be written as a polynomial

$$
V_{s}=\sum_{k=1}^{2^{s}} a_{k} t_{s}^{k}
$$

with coefficients $a_{k}$ in $V_{s-1} \subset \Delta_{s-1}$; further, $a_{1}=V_{1} V_{2} \cdots V_{s-1}$ is invertible in $\Delta_{s-1}$. It follows from Lemma 9.1 that $V_{s}$ is invertible in $\Delta_{s-1}\left[\left[t_{s}\right]\right]^{\prime}$. Consequently there is a unique inclusion $i_{s}$ of $\Delta_{s-1}$-algebras from $\Delta_{s}=\Delta_{s-1}\left[V_{s}^{ \pm}\right]$into $\Delta_{s-1}\left[\left[t_{s}\right]\right]^{\prime}$ that sends $V_{s}$ to the polynomial (9.2). We compose this inclusion with the residue map of (9.1) to obtain Res: $\Delta_{s} \rightarrow \Delta_{s-1}$ :

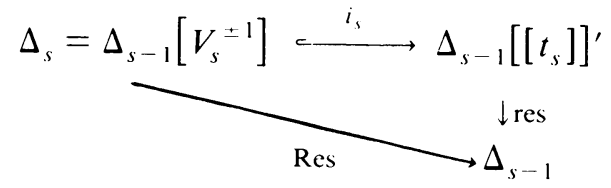


The main result of this section is

Proposition 9.2. Let $\mathrm{\partial}: \Delta_{s} \rightarrow \Delta_{s-1}$ be as given in (8.2). Then $\mathrm{\partial}=\operatorname{Res}: \Delta_{s} \rightarrow \Delta_{s-1}$.

Proof. Induct on $s$. If $s=1$ it is clear. Suppose the result proved for $s-1$, for some $s \geqslant 2$. Note that $\Delta_{s-1}$ is a subalgebra of $\Delta_{s}$, so that both $\Delta_{s-1}$ and $\Delta_{s}$ can be regarded as modules over $\Delta_{s-1}$. One sees easily that both Res and $\partial$ are $\Delta_{s-1}$-linear maps, so it suffices to check that Res and $\partial$ agree on the elements $\left\{v_{s}^{n} \mid-\infty<n<\infty\right\}$ which form a $\Delta_{s-1}$ basis for $\Delta_{s}$. To this end, define an algebra homomorphism $\alpha$ : $\Phi_{s-1} \rightarrow \Phi_{s}$ (with $\Phi_{s}$ defined as in (2.7)) by

$$
\alpha\left(t_{j}\right)=t_{j+1}+t_{j+1}^{2} / t_{1}
$$

It is easy to check that $\alpha\left(v_{j}\right)=v_{j+1}$ for all $j, 1 \leqslant j \leqslant s-1$. In particular, $\alpha\left(\Delta_{s-1}\right) \subseteq$ $\Delta_{s}$. We can define an algebra homomorphism $\alpha^{\prime}: \Delta_{s-2}\left[\left[t_{s-1}\right]\right]^{\prime} \rightarrow \Delta_{s-1}\left[\left[t_{s}\right]\right]^{\prime}$ in such a way that the following diagram commutes:

$$
\begin{array}{ccccc}
\Delta_{s-1} & \stackrel{i_{s-1}}{\rightarrow} & \Delta_{s-2}\left[\left[t_{s-1}\right]\right]^{\prime} & \stackrel{\text { res }}{\rightarrow} & \Delta_{s-2} \\
\alpha \downarrow & & \downarrow \alpha^{\prime} & & \downarrow \alpha \\
\Delta_{s} & \stackrel{i_{s}}{\rightarrow} & \Delta_{s-1}\left[\left[t_{s}\right]\right]^{\prime} & \stackrel{\text { res }}{\rightarrow} & \Delta_{s-1}
\end{array}
$$

In fact, the left-hand square commutes if we set $\alpha^{\prime}\left(v_{j}\right)=v_{j+1}(1 \leqslant j \leqslant s-2)$ and $\alpha^{\prime}\left(t_{s-1}\right)=t_{s}\left(1+t_{s} / v_{1}\right)$. So we take this as the definition. In particular,

$$
\alpha^{\prime}\left(t_{s-1}^{k}\right)=t_{s}^{k} \sum_{j=0}^{\infty}\left(\begin{array}{l}
k \\
j
\end{array}\right)\left(t_{s} / v_{1}\right)^{j}
$$

for $-\infty<k<\infty$, where we interpret the binomial coefficients as in [8, §2]. Now the commutativity of the right-hand square in (9.4) follows from (9.5), since $\left(\begin{array}{c}k \\ -k-1\end{array}\right)$ is nonzero modulo 2 if and only if $k=-1$. But now we can use (9.4) to prove that $\partial\left(v_{s}^{n}\right)=\operatorname{Res}\left(v_{s}^{n}\right)$ for all integers $n$. In fact, (9.4) implies

$$
\begin{aligned}
\operatorname{Res}\left(v_{s}^{n}\right) & =\operatorname{res} \circ i_{s} \circ \alpha\left(v_{s-1}^{n}\right)=\alpha \circ \text { res } \circ i_{s-1}\left(v_{s-1}^{n}\right) \\
& =\alpha \circ \operatorname{Res}\left(v_{s-1}^{n}\right)
\end{aligned}
$$

where we have used the definition (9.3). But by the inductive hypothesis $\operatorname{Res}\left(v_{s-1}^{-1}\right)$ $=1 ; \operatorname{Res}\left(v_{s-1}^{n}\right)=0$ if $n \neq-1$. So (9.6) gives $\operatorname{Res}\left(v_{s}^{-1}\right)=1 ; \operatorname{Res}\left(v_{s}^{n}\right)=0$ if $n \neq-1$. So $\operatorname{Res}\left(v_{s}^{n}\right)=\partial\left(v_{s}^{n}\right)$, and we are done.

This last result implies Theorem 1.4 of the Introduction.

\section{BIBLIOGRAPHY}

1. A. K. Bousfield, E. B. Curtis, D. M. Kan, D. G. Quillen, D. L. Rector and J. W. Schlesinger, The mod-p lower central series and the Adams spectral sequence. Topology 5 (1966), 331-342.

2. A. K. Bousfield and E. B. Curtis, A spectral sequence for the homotopy of nice spaces, Trans. Amer. Math. Soc. 151 (1970), 457-479.

3. L. E. Dickson, $A$ fundamental system of invariants of the general modular linear group with a solution of the form problem. Trans. Amer. Math. Soc. 12 (1911), 75-98.

4. L. Kristensen, A Cartan formula for secondary cohomology operations, Math. Scand. 16 (1965), $97-115$.

5. I. Madsen, On the action of the Dyer-Lashof algebra in $H_{*}(G)$, Pacific J. Math. 60 (1975), 235-275. 
6. J. W. Milnor and J. C. Moore, On the structure of Hopf algebras, Ann. of Math. (2) 81 (1965), 211-264.

7. H. Mui, Modular invariant theory and the cohomologv algebras of symmetric groups, J. Fac. Sci. Univ. Tokyo Sect. IA Math. 22 (1975), 319-369.

8. W. M. Singer, A new chain complex for the homology of the Steenrod algebra, Proc. Cambridge Philos. Soc. 90 (1981), 279-292.

9. Iterated loop functors and the homology of the Steenrod algebra. II: A chain complex for $\Omega_{s}^{h} M$, J. Pure Appl. Algebra 16 (1980), 85-97.

10. The construction of certain algebras over the Steenrod algebra, J. Pure Appl. Algebra 11 (1977), 53-59.

11. C. Wilkerson, Classifying spaces, Steenrod operations, and algebraic closure, Topology 16 (1977), $227-237$.

Department of Mathematics, Fordham University, BronX, New York 10458 